\section{§1. Study on TAE-Induced Fast-Ion Loss Process in LHD}

Ogawa, K., Isobe, M., Toi, K., Shimizu, A., Spong, D.A. (Oak Ridge National Laboratory), Osakabe, M., Yamamoto, S. (Kyoto Univ.), LHD Experiment Group

Much attention has been given to the effects of fastion-driven MHD instabilities such as toroidal-Alfvén eigenmodes (TAEs) on fast-ion transport and/or loss in magnetically confined fusion because those instabilities can potentially induce anomalous fast-ion losses. In Large Helical Device (LHD), recurrent bursts of TAEs have been often excited by super-Alfvénic ions produced by highenergy neutral beam (NB) injection, leading to anomalous fast-ion losses. A Mirnov coil array indicates that TAEs observed in LHD have a mode structure of $m / n=\sim 1 / 1$ and are characterized by a relatively wide radial profile ${ }^{1)}$.

Measurements of fast-ion losses induced by these TAE instabilities are conducted in NB-heated LHD plasmas having three magnetic axis positions at finite $\beta$, i.e. $R_{\text {mag }}=3.75 \mathrm{~m}$ (case A), $3.86 \mathrm{~m}$ (case B), and $4.00 \mathrm{~m}$ (case C). As $R_{\text {mag }}$ becomes larger, fast-ion orbits tend to deviate largely from magnetic flux surfaces as shown in Fig. 1 (a). In this paper, $r / a$ and $B_{\mathrm{t}}$ represent normalized minor radius and toroidal magnetic field strength, respectively. Note that the TAE gap becomes wider with larger $R_{\text {mag }}$ compared with smaller $R_{\text {mag }}$ since magnetic shear in LHD becomes weaker as $R_{\text {mag }}$ becomes larger. Figure 1 (c) shows an increment of fast-ion loss flux due to the TAEs from the neoclassical orbit loss level $\left(\Delta \Gamma_{\text {fast ion }}\right)$ at the SLIP position normalized by fast-ion populations created by co-injected NB, i.e. $P_{\mathrm{NBco}} \times \tau_{\mathrm{s}}$ as a function of $b_{\theta \mathrm{TAE}} / B_{\mathrm{t}}$. Here, $P_{\mathrm{NBco}}, \tau_{\mathrm{s}}$, and $b_{\theta \mathrm{TAE}}$ stand for co-injected NB power, the Spitzer slowing-down time, and poloidal magnetic fluctuation amplitude at the Mirnov coil position placed on the vacuum vessel, respectively. In case $\mathrm{B}$, the dependence of the fast-ion loss flux on $b_{\theta \mathrm{TAE}} / B_{\mathrm{t}}$ changes at $b_{\theta \mathrm{TAE}} / B_{\mathrm{t}} \sim 7 \times 10^{-5}$. In the low $b_{\theta \mathrm{TAE}}$ regime, $\Delta \Gamma_{\text {fast }}$ ion is proportional to $b_{\theta \mathrm{TAE}}$ whereas it scales as $\Delta \Gamma_{\text {fast ion }} \propto$ $b_{\theta \mathrm{TAE}}{ }^{2}$ in the higher $b_{\theta \mathrm{TAE}}$ regime. According to a theory ${ }^{2)}$, $\Delta \Gamma_{\text {fast ion }}$ proportional to $b_{\theta \mathrm{TAE}}$ is suggested to be due to a convective type loss process whereas $\Delta \Gamma_{\text {fast ion }}$ scaling as the square of $b_{\theta \mathrm{TAE}}$ is suggested to be due to a diffusive type loss process. The experimental result indicates that the fastion loss process changes from convective to diffusive in case B. On the other hand, in cases $A$ and $C$, this change of loss processes has not been observed for these $b_{\theta \mathrm{TAE}} / B_{\mathrm{t}}$ ranges although the change may appear in unexplored regions.

Previous work modeling for axisymmetric tokamak predicts that the process of TAE-induced fast-ion transport changes from a convective type to a diffusive type according to $b_{\theta \mathrm{TAE}}{ }^{3)}$. To study fast-ion loss processes in a three-dimensional helical configuration precisely, simulations based on an orbit following model, DELTA5D ${ }^{4)}$, have been performed. TAE magnetic fluctuation is modeled

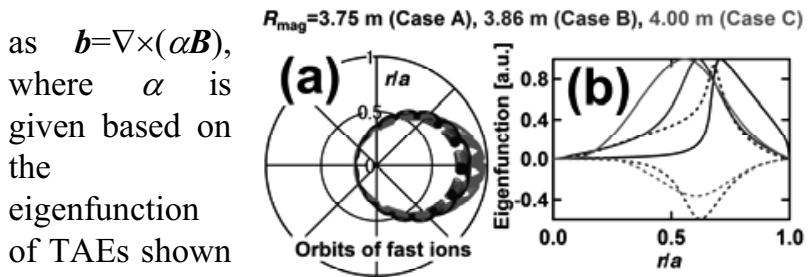

in Fig. 1 (b).

The

eigenfunction is calculated by an ideal MHD calculation code treating shear-Alfvén waves, AE3D ${ }^{5}$. The

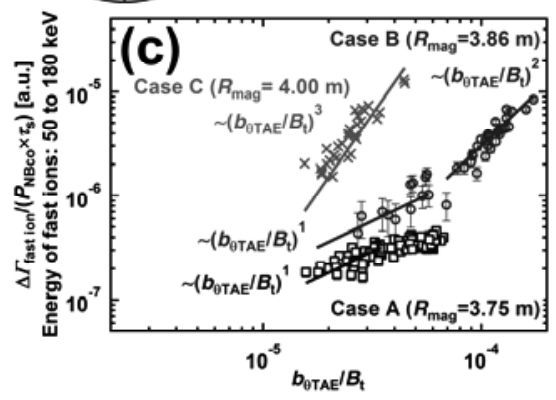

dependence of Fig. 1 (a) Co-circulating fast-ion orbits in $\Delta \Gamma_{\text {fast }}$ ion $/\left(P_{\mathrm{NBco}} \times \tau_{\mathrm{s}}\right)$ on $b_{\theta \mathrm{TAE}} / B_{\mathrm{t}}$ obtained simulation is $b_{\theta \mathrm{TAE}} / B_{\mathrm{t}}$ changes at $b_{\theta \mathrm{TAE}} / B_{\mathrm{t}} \sim 7 \times 10^{-5}$ in cases $\mathrm{A}, \mathrm{B}$, and $\mathrm{C}$ on $B_{\mathrm{t}}=0.6 \mathrm{~T}$. (b) Eigenfunctions of TAE calculated by AE3D for cases $\mathrm{A}, \mathrm{B}$, and $\mathrm{C}$. (c) $\Delta \Gamma_{\text {fast }}$ ion $/\left(P_{\mathrm{NBco}} \times \tau_{\mathrm{s}}\right)$ as a function of $b_{\theta \mathrm{TAE}} / B_{\mathrm{t}}$. shown in Fig. 2. case B.

In case $A$, the

calculated dependence is similar to Fig. 1 (c) in the low $b_{\theta \mathrm{TAE}}$ regime. The change of the loss process to a diffusive nature appears at $b_{\theta \mathrm{TAE}} / B_{\mathrm{t}}$ of $\sim 10^{-4}$ that is in unexplored regions of experiments. In case $B$, the change of the loss process from a convective type to a diffusive type is successfully reproduced. As described in Ref. 3, our calculation suggests that with a convective type loss process, the barely confined fast ions near the confinement/loss boundary are lost. On the other hand, the fast ions confined in the interior region of the plasma are lost with a diffusive type loss process. Experimentally observed phenomena are explained as follows. In the small $b_{\theta \mathrm{TAE}}$ region, the convective type loss is dominant. As $b_{\theta \mathrm{TAE}}$ increases, the diffusive type loss increases and exceeds the convective type loss at a certain $b_{\theta \mathrm{TAE}}$ level.

1) Toi, K. et al. : Plasma Phys. Control. Fusion $\mathbf{5 3}$ (2011) 024008.

2) Heidbrink, W.W. et al., Phys. Fluids B 5 (1993) 2176.

3) Sigmar, D. et al. : Phys. Fluids B 4 (1992) 1506.

4) Spong, D. A. : Phys. Plasmas 18 (2011) 056109.

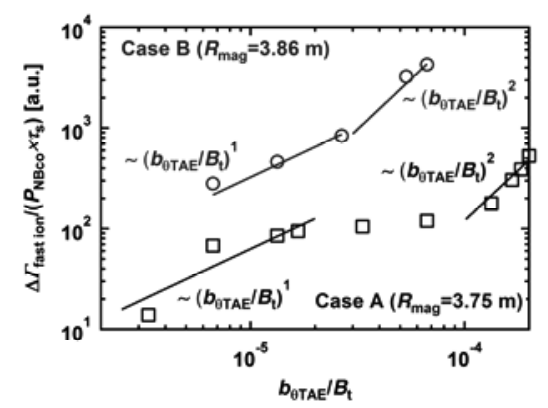

Fig. $2 \Delta \Gamma_{\text {fast ion }} /\left(P_{\mathrm{NBco}} \times \tau_{\mathrm{s}}\right)$ as a function of $b_{\theta \mathrm{TAE}} / B_{\mathrm{t}}$ in calculations for cases $\mathrm{A}$ and $B$. The dependence is similar to that obtained in experiments in case A process from a convective type 17 (2010) 022106. simulation for case B. 